\title{
Prediction on Hourly Cooling Load of Buildings Based on Neural Networks
}

\author{
Hu Chaowen and Wei Dong \\ School of Electrical and Information Engineering, Beijing University of Civil \\ Engineering and Architecture, Beijing 100044 \\ 1256788751@qq.com,weidong_999@163.com
}

\begin{abstract}
Energy conservation and indoor environment concerns have motivated extensive research on various aspects of control of Heating, Ventilating and Air-Conditioning (HVAC) and building systems. The study on optimal operation as well as modeling of HVAC and building systems is one of the fastest growing fields that contribute to saving energy and improving indoor environment of buildings. The reasonable operation adjustment is one of the main methods to improve the energy efficiency. Cooling load prediction is the foundation of the optimization operation. This paper is devoted to the development of a comprehensive modeling of cooling load for a large building with ice-storage systems in Beijing, China. The models describe the dynamics of cooling load, outdoor climate parameters and indoor parameters as one multi-variable nonlinear system in a way that is useful for prediction analysis. The cooling load data collected is from June to September, and then the method of similarity for both longitudinal and transverse waveforms is used to judge whether there is abnormal data. The optimal parameter setting in the proposed model is studied. Principle Component Analysis (PCA) method was applied to select input parameters. A load prediction model has been constructed based on BP neural networks. Taking account of the generalization ability of neural networks, this paper has chosen the bayesian regularization algorithm, which can get better fitting effect than other training algorithms, to train the neural networks. Then, the BP neural network model is used for the summer hourly cooling load prediction of the business building. Evaluation of the prediction accuracy of the proposed models is based on the root mean square error (RMSE). The results show that the prediction model can accurately predict the future hourly load of 1 week and 1 day, with the prediction error at $1.60 \%$ and $1.18 \%$ respectively. The analysis shows that this model is suitable for the practical engineering application and can provide a basis for optimal operation of air conditioning control systems of large public buildings.
\end{abstract}

Keywords: neural networks, air-conditioning systems, load prediction, bayesian regularization

\section{Introduction}

Accelerated urbanization and continuous improvements in living standards have resulted in an increasing demand on energy used in buildings. In china, energy consumption for buildings accounts for about $40 \%$ of the total energy use ${ }^{[1]}$. HVAC systems are one of the critical systems used in modern buildings and account for approximately $60 \%$ of the total energy consumption in a typical building ${ }^{[2]}$. As a result, the prediction on cooling load of air conditioning systems in buildings is significant for improving their energy performance, with the aim of achieving energy conservation and reducing environmental impact.

For air conditioning systems, one of the main methods to improve energy efficiency is reasonable operations including adjusting the starting time of cooling to meet start-up 
loads, minimizing or limiting the electric on-peak demand, optimizing the costs and energy utilization in cool storage systems, and related energy and cost needs in other HVAC systems ${ }^{[3-5]}$. Load prediction forms the foundation for optimizing the operations of air conditioning systems. However, accurately predicting the building cooling load is a challenging work. Cooling load in buildings is affected by many factors, which are quite complex, and they can be grouped into two main categories: the meteorological data and the optical and thermal properties of buildings.

In recent years, the artificial neural networks(ANNs), a kind of intelligent bionic model, have been widely concerned, and their practical applications in many fields have gained remarkable results. ANNs have represented a new paradigm in the load prediction. They work as a black-box model. Thus, it is not necessary to have detailed information about the system because ANNs learn the relationship between input and output parameters by means of historical data. Another advantage is the ability to manage large complex systems with many parameters interrelated between themselves, since they may ignore the excess of information with minor importance.

In this paper, a building cooling load prediction model based on BP neural networks is developed at first. And the optimal parameter setting in the proposed model is studied. Then, the BP neural network model is used for the summer hourly cooling load prediction of a business building in Beijing, China.

\section{Related Work}

At present, forecasting methods can be generally divided into two broad categories ${ }^{[6]}$ : qualitative and quantitative prediction. Qualitative methods are also known as intuitive prediction. Quantitative forecasting methods are similar to statistical prediction. For applications in the field of HVAC systems, quantitative predictions are often used and mathematical methods and statistics along with probability theory are applied to collect and deal with large amount of historical data ${ }^{[7]}$. In the HVAC field, how to predict the building cooling load accurately attracts researchers' attention all the way ${ }^{[8-15]}$. Several prediction methods have ever been adopted, such as the Admittance and Fourier methods ${ }^{[16]}$, the correlation method ${ }^{[17,18]}$, the transfer function method ${ }^{[19]}$, the Monte Carlo simulation method ${ }^{[20]}$, and so on.

Regression analysis ${ }^{[21,22]}$, time series analysis ${ }^{[21,23]}$ and "gray box" theory ${ }^{[24]}$ are traditional load prediction methods, and to use these methods requires the establishment of accurate mathematical models of different kinds of buildings. However, the cooling load prediction is a difficult task due to the complexity in the time series of load, which presents a high non-linearity among the factors, as well as seasonality. And in practical engineering, it is very difficult to quantitatively describe or measure all influencing factors very well during the whole building prediction process. Therefore, when using these traditional methods to forecast the load of air conditioning systems, not only the process of establishing model is rather complicated, but also the accuracy of the results is often not satisfied.

The ANN method is convenient, and fits for an ordinary operator to implement after the model has been established. The practical success of ANNs can be attributed to their distinctive features: learning, self-adaptative, fault tolerance, flexibility and real time response ${ }^{[25-27]}$. What is more, ANNs are widely accepted as a technology offering an alternative way to tackle complex and ill-defined problems because of their strong nonlinear mapping ability. They are especially good at capturing the non-linear relationships and changing trends of the building load. Neural network models can realize any nonlinear mapping between the input and output, and there is no need to know the mathematical equation describing the load and the influence factors in advance. Hence it has been popularly applied to predict the building cooling load ${ }^{[28-34]}$ and building energy consumption $^{[35,36]}$. 


\section{Neural Network Model}

In this paper a multiple input - single output load prediction model has been constructed based on BP neural networks. The core methodology used for the ANNs forecasting model construction could include data collection, input parameters selection, neural network construction, data preprocessed and neural network training.

\subsection{BP Neural Network}

As for the artificial neural networks, the most used one in engineering applications is BP (Back Propagation) neural networks, which consist of an input layer, one or more hidden layers and one output layer. In its simplest version, each neuron is connected to another neurons from a previous layer through adaptable synaptic weights, and it is in this set of connection weights where the network knowledge is stored.

In this study, three-layered BP neural networks is used. The input layer receives information from the external sources and passes this information to the network for processing. The hidden layer receives information from the input layer, and does all the information processing, and the output layer receives processed information from the network, and sends the results out to an external receptor.

Output from a neuron in the input layer is shown as Eq. (1)

$\mathrm{O}_{\mathrm{ij}}=\mathrm{X}_{\mathrm{ij}}, \quad \mathrm{i}=1,2, \ldots, \mathrm{N}, \quad \mathrm{j}=1,2, \ldots, 6$

Output from a neuron in the hidden layer is shown as Eq. (2)

$\mathrm{O}_{\mathrm{ik}}=\mathrm{f}\left(\sum_{\mathrm{j}=0}^{6} \mathrm{~W}_{\mathrm{kj}} \mathrm{O}_{\mathrm{ij}}\right), \mathrm{k}=1,2, \ldots, \mathrm{m}$

Output from a neuron in the output layer is shown as Eq. (3)

$$
\mathrm{Y}_{\mathrm{i}}=\mathrm{f}\left(\sum_{\mathrm{k}=0}^{\mathrm{m}} \mathrm{W}_{\mathrm{k}} \mathrm{O}_{\mathrm{ik}}\right)
$$

where, $\mathrm{m}$ is the hidden neurons' number; $\mathrm{W}_{k j}$ and $\mathrm{W}_{k}$ are the connection weight from the jth input neuron to the kth hidden neuron, and that from kth hidden neuron to the output neuron, respectively; $\mathrm{f}(\mathrm{)})$ is the sigmoid transfer function given by Eq. (4)

$\mathrm{f}(\mathrm{x})=\frac{1}{1+\mathrm{e}^{-\mathrm{x}}}$

The $\mathrm{BP}$ algorithm is designed to solve the problem of determining the connection weight values. Through training, the non-linear relationship between the buildings cooling loads and their correlative influence parameters is established.

\subsection{Data Collection}

In order to obtain an appropriate neural network model, a set of historic data acquired at the building has been used. More specifically, the historic data set is composed by 2928 points from 06/01/2007 to 09/30/2007, with load reports generated at 1-hour intervals, $24 \mathrm{~h}$ a day. In addition, the historic data has been split into three different data-sets to train, validate and test the neural network model. The first data set is the Training Data Set and it is used for obtaining the ANNs parameters. The second one is the Validation Data Set, which is necessary in the training and post-training processes in order to prevent the over-training in the ANNs. The final data set is the Test Data Set, whose objective is to provide an independent data set for benchmarking and testing purposes.

$\operatorname{DeST}^{[37]}$ is used to calculate the business building's hourly cooling loads, which are taken as the basic values to compare with the predicted values from the BP model. DeST is a building environment and energy consumption simulation software developed by Tsinghua University. It adopts the state space method for the load simulation ${ }^{[38]}$. Stage design and simulation are its basic features ${ }^{[39]}$. Its reliability has ever been proven by 
analytical test, inter-model comparison and experiment results ${ }^{[40]}$. In the past 10 years, DeST has played an important role in the prediction of building energy consumption in many projects ${ }^{[41-43]}$. By collecting data from the flow meters and temperature sensors, the total volume flow of chilled water and the system total return/supply water temperatures are known. Therefore, the real-time cooling load of air-conditioning system can be calculated by equation (5).

load $=\mathrm{Q} \times \mathrm{p} \times \mathrm{C}\left(\mathrm{T}_{2}-\mathrm{T}_{1}\right)$

where load: the real-time cooling load, $103 \mathrm{Kal} / \mathrm{h}$;

$Q$ :the total chilled water volume flow of air-conditioning system, $\mathrm{m}^{3} / \mathrm{h}$; $p$ :the density of water, $\mathrm{Kg} / \mathrm{m}^{3}$;

$\boldsymbol{C}$ :the specific heat capacity of water, $\mathrm{Kal} / \mathrm{Kg} \cdot{ }^{\circ} \mathrm{C}$;

$\boldsymbol{T}_{2}$ :the return water temperature of air-conditioning system, ${ }^{\circ} \mathrm{C}$;

$\boldsymbol{T}_{\boldsymbol{I}}$ : the supply water temperature of air-conditioning system, ${ }^{\circ} \mathrm{C}$.

\subsection{ANN Model Inputs Selection}

When the construction of a building is complete, the envelope and construction material are fixed, and the most important parameters affecting cooling load are the outdoor climate parameters and indoor change of occupancy, starting and stopping of the equipments. The selection of input parameters has a critical role in the ANN modeling. To predict the cooling load for an air-conditioning system, the potential input parameters will be the following ones:

1.outdoor temperature;

2.atmospheric pressure;

3.cloud state;

4.cloud cover (0-10 levels);

5.relative humidity;

6.outdoor air enthalpy;

7.outdoor air density;

8.wind speed;

9. wind direction;

10.intensity of solar radiation;

11.indoor load;

12.indoor equipment heat load.

Theoretically combed, any factors that impact the cooling loads can be used as input parameters of neural network model. Generally speaking, the nonlinear mapping ability of neural networks will be stronger with much more input information, and the prediction accuracy will be higher. However, it will increase the computer solving time, and also increase the difficulty of control system and the initial investment due to the added measurement points and sensors. Meanwhile, in the practical engineering applications, a lot of input parameters cannot be often available.

There is a group of independent parameters, and the selection of inputs has to be done in order to avoid the use of parameters with few relevance in the prediction and, at the same time, including the most relevant ones. One of the distinguished methods for feature selection could be Principle Component Analysis (PCA) methods, which directly apply on the data without the need to build and test models. After several data analysis techniques, the initial list of potential parameters has been reduced to the following ones:

1. outdoor temperature $\left({ }^{\circ} \mathrm{C}\right)$;

2. relative humidity $(\%)$;

3. intensity of solar radiation $\left(\mathrm{W} / \mathrm{m}^{2}\right)$;

4. speed and direction of the wind $(\mathrm{m} / \mathrm{s})$;

5. indoor electrical load $\left(\mathrm{W} / \mathrm{m}^{2}\right)$. 
3.3.1. Outdoor Temperature and Relative Humidity: Outdoor temperature is the primary factor, which greatly decides the cooling load of an air conditioning system. Meanwhile, the outdoor temperature presents positive correlations with the hourly load. Relative humidity is also one of the main external disturbance factors affecting the cooling load, and it is shown as the quantity of latent heat. Hence, this paper takes both outdoor temperature and relative humidity as the input parameters of the building cooling load prediction model.

3.3.2. Intensity of Solar Radiation: Solar radiation is the most basic and important energy on earth, and the distribution and changes of it play a vital role in the formation and changes of climate. Solar radiation is the main factor causing the outdoor temperature changing, and its influence on the cooling load is larger. Therefore, this paper chooses the intensity of solar radiation as an input parameter.

3.3.3. Speed and Direction of the Wind: The wind direction refers to the direction of the wind blowing. Meteorological offices commonly use eight directions to release the forecast of the wind, namely the east (E), south (S), west (W), north (N), southeast (SE), southwest $(\mathrm{SW})$, northwest $(\mathrm{NW})$ and northeast (NE).Wind direction observation is represented with 16 bearing, that is, among the eight azimuth add north-northeast (NNE), east-northeast (ENE), east-southeast (ESE), south-southeast (SSE),south-southwest (SSW),west-southwest (WSW), west-northwest (WNW) and north-northwest (NNW).Actually, wind report also often use Arabic numerals to show the wind direction within the scope of $0^{\circ} \sim 360^{\circ} .0^{\circ}$ is on behalf of the north, $90^{\circ}$ is on behalf of the east, $180^{\circ}$ is on behalf of the south, and $270^{\circ}$ is on behalf of the west, and the rest is deduced. Sometimes, such names "PianNanFeng", "PianBeiFeng" are used in the weather forecast, and "Pian" said that the wind moved around a certain position for a small range.The 16 wind direction is shown in figure 1 .

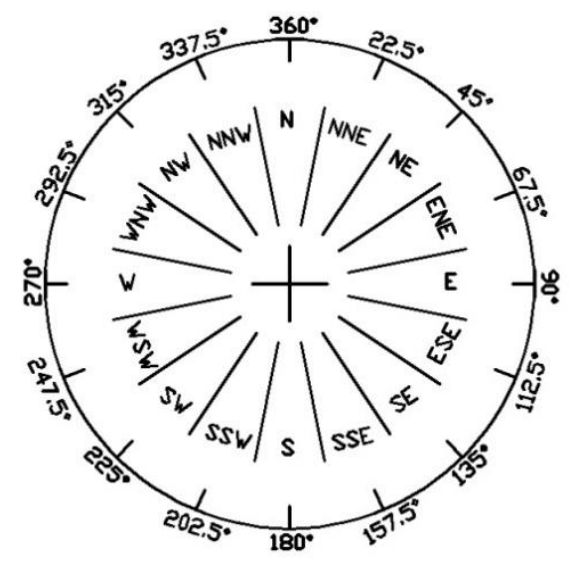

Figure 1. The 16 Wind Direction

3.3.4. Indoor Electrical Load: In addition to the above meteorological data, internal disturbance factors account for a large proportion in the air conditioning load. Thus the internal disturbance is considered as one of the input variables. Because of lack of actual measurement data, the experience value of indoor electric load was used, and more specifically, the experience value takes $40 \mathrm{w} / \mathrm{m}^{2}$. The indoor total electrical load of working hours is 0.14957 million $\mathrm{m}^{2} \mathrm{x} 40 \mathrm{~W} / \mathrm{m}^{2}$, and the non-working time for $20 \%$ of working time. 


\subsection{Data Preprocessed}

Because the actual measurement data are easily affected by the error of collecting instruments, and the instability of monitoring platform may also lead to the data error or bias, in order to eliminate abnormal data, the pretreatment to the actual measurement data must be carried on. In this paper, the method of similarity ${ }^{[44]}$ for both longitudinal and transverse waveforms of air conditioning system operation parameters is used to judge whether there is abnormal data. Longitudinal similarity refers that the operation parameters of similar time generally have not particularly big mutation, and have smoothness. Transverse similarity refers that the peak and valley for the same type of daily operation parameters curve have the same time in the adjacent days, and the curve shapes are similar. Therefore, for those data compared with its adjacent two sets of data or the same time data of two days before and after, if the relative error is more than the engineering threshold(generally 150\%), the data is considered as abnormal data. For abnormal data, the general approach is to eliminate it directly, and then use the average of reliable data instead.

In the training stage for ANN, in order to improve the calculation efficiency, and prevent individual data from overflowing during the calculation, it need to normalize ${ }^{[45]}$ the input data and the known corresponding output values, namely processing the data into numerical value between $0 \sim 1$. Assuming that the original input and output value of the neural network is xdi and ydi, after the normalization processing they become

$x_{i}=\frac{\left(x_{d i}-x_{d \min }\right)}{\left(x_{d \max }-x_{d \min }\right)}$

and

$\mathrm{t}_{\mathrm{i}}=\frac{\left(\mathrm{y}_{\mathrm{di}}-\mathrm{y}_{\mathrm{dmin}}\right)}{\left(\mathrm{y}_{\mathrm{dmax}}-\mathrm{y}_{\mathrm{dmin}}\right)}$

where, $\mathrm{x}_{i}$ is the normalized neural network input value; $\mathrm{x}_{d i}$ is the original input value; $\mathrm{x}_{d m i n}$ is the minimum of the original input values; $\mathrm{x}_{d \max }$ is the maximum of the original input values; $\mathrm{t}_{i}$ is the normalized neural network target value(teacher value); $\mathrm{y}_{d i}$ is the original target value; $\mathrm{y}_{\text {dmin }}$ is the minimum of the original target values; $\mathrm{y}_{\text {dmax }}$ is the maximum of the original target values.

After training, the neural network gets the predicted value oi from BP model, which range from 0 to 1 . Then, it should be de-normalized into the actual prediction value by Eq. (8).

$\mathrm{y}_{\mathrm{pi}}=\mathrm{y}_{\mathrm{dmin}}+\mathrm{o}_{\mathrm{i}}\left(\mathrm{y}_{\mathrm{dmax}}-\mathrm{y}_{\mathrm{dmin}}\right)$

where, $\mathrm{y}_{p i}$ is the de-normalized value of the predictive value; $\mathrm{o}_{i}$ is the predicted output value of the neural network.

\subsection{Evaluation Indices}

Suppose that all the normalized input parameters compose a vector Xi (i represents one input sample), and $\mathrm{Yi}$ is the normalized building cooling load under the input sample i. When the total number of samples is $\mathrm{N}$, the sample set is defined as $\{(\mathrm{Xi}, \mathrm{Yi})\}_{\mathrm{i}=1}^{\mathrm{N}}$.

The performance evaluation indices to BP neural network models adopted throughout this paper is the root mean square error (RMSE), which is defined as follows:

$$
\text { RMSE }=\sqrt{\frac{1}{\mathrm{~N}} \sum_{\mathrm{i}=1}^{\mathrm{N}}\left(\frac{\mathrm{q}_{\mathrm{i}}-\widehat{\mathrm{q}_{1}}}{\mathrm{q}_{1}}\right)^{2}} \times 100 \%
$$

where $\widehat{q}_{1}$ is the predicted value of $q_{i}$.

\subsection{Neural Network Construction}

After the input parameters selection, the hourly building cooling load chosen as the model's output, a BP neural network cooling load prediction model is established. A limited number of hidden layer is enough to solve the problem of nonlinear and hysteresis, and three-layered neural network has the ability to approximate any functions, 
including non-linear function ${ }^{[46]}$. In this study, three-layered BP neural network is used, consisting of an input layer, a hidden layer and an output layer. The number of neurons in a hidden layer drastically affects the outcome of the network training. It must be sufficient for correct modeling of the problem, and on the other hand, it should be low to ensure generalization. However there is no general rule for selecting the number in a hidden laye ${ }^{[47]}$, but only experience and trial \& error method. So, experience and a large number of experiments are usually relied on for practical guidance.

The optimal number of neurons in a hidden layer is not known beforehand. Hence, some kind of model selection (parameter search) must be done. The goal is to identify optimal number of neurons in a hidden layer so that the model can accurately predict the unknown data. By constantly trying to change the number of hidden layer nodes, the convergence speed and the ability to fit the model are improved. This process will not be stopped until the network output error meets the requirement. Considering the input neurons' number of six, and the output neuron's number of one, this paper explores the hidden layer neurons' number in the range of $10 \sim 18$ using stepwise searching method, and finds that the optimal number is 16.

More specifically, for these nine cases, BP model with input parameters determined above is established. And the established BP model is used to predict the hourly building cooling load for the next 1 week and 1 day. The RMSE of the training sample under various cases are compared, which is shown in Table 1. From Table 1, we can see there is the same lowest RMSE point in terms of both 1 week and 1 day cooling load prediction. From these results, we can choose the number of neurons in the hidden layer to be 16 , considering the accuracy.

Table 1. The Prediction Errors of BP Model in Different Cases (\%)

\begin{tabular}{c|lc}
\hline $\begin{array}{l}\text { Case (the number of neurons } \\
\text { in the hidden layer) }\end{array}$ & $\begin{array}{l}1 \text { Week } \\
\text { RMSE }\end{array}$ & $\begin{array}{c}1 \text { Day } \\
\text { RMSE }\end{array}$ \\
\hline 10 & 0.0185 & 0.0132 \\
\hline 11 & 0.0196 & 0.0150 \\
\hline 12 & 0.0188 & 0.0143 \\
\hline 13 & 0.0168 & 0.0145 \\
\hline 14 & 0.0173 & 0.0148 \\
\hline 15 & 0.0169 & 0.0131 \\
\hline 16 & 0.0160 & 0.0118 \\
\hline 17 & 0.0213 & 0.0122 \\
\hline 18 & 0.0257 & 0.0133 \\
\hline \hline
\end{tabular}

Because the wind direction is not the digital quantity parameter. In order to reduce the neural network training error, there will be wind speed vector toward two direction(the north and the east)respectively for projection. After projection, the wind speed(North) and wind speed (East) will be regarded as two inputs of the network instead of the original wind speed and direction. The topology of the three-layered ANN model for hourly load prediction is shown in figure 2 . 


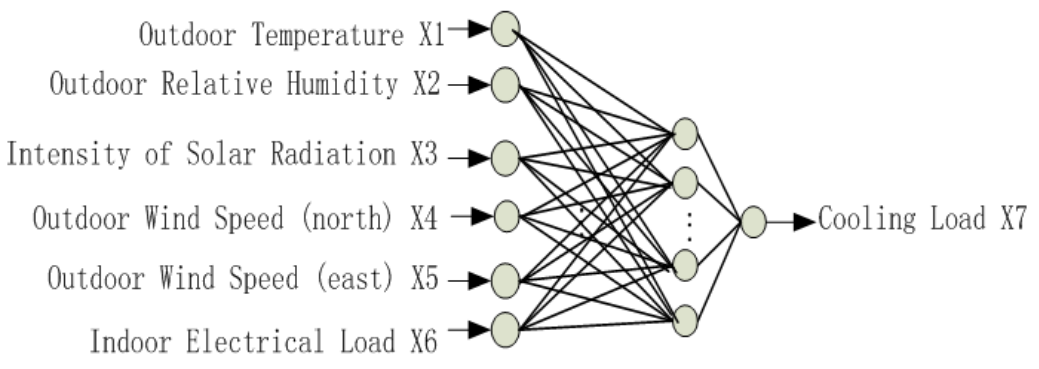

Figure 2. The Topology of Three Layer Neural Network

\subsection{Neural Network Training}

Training is the process to calibrate the connection weights using an adequate learning method. For the standard BP neural network algorithm, there exist a few shortcomings such as being easy to fall into a local minimum point, slow learning and convergence speed, difficulty to determine the network topology structure, overtraining and so on ${ }^{[48,49]}$. For this reason, researchers put forward many improved algorithms to speed up the network training speed and avoid falling into a local minimum value. The steepest descent $\mathrm{BP}$ algorithm, the elasticity BP algorithm, the momentum BP algorithm, the variable gradient BP algorithm, Newton algorithm, L-M ( Levenberg - Marquardt )algorithm and bayesian regularization methods are part of the improved algorithms. For specific issues, choosing what kind of algorithm depends on many factors. In general, for the small or medium-sized neural network, which contains hundreds of network weights, the appropriate one is L-M algorithm, who has the fastest convergence speed ${ }^{[50]}$ and a smaller mean square error (MSE), while the bayesian regularization algorithm can effectively improve the generalization ability of neural network ${ }^{[51]}$.

The nature of neural network modeling is to find out the relationship(the mapping relations between the inputs and outputs), which is included in limited sample data. Then the neural network can give appropriate output for untrained input, which is known as the function of generalization ${ }^{[52]}$.The generalization ability of BP neural network based on the above algorithms (except for the bayesian regularization algorithm) did not achieve the ideal effect, while the generalization ability is the important symbol to measure whether the performance of neural network is good or not. So, this paper uses Bayesian regularization ${ }^{[52,53]}$ algorithm to train BP network load prediction model.

With the regularization method the performance index is modified to include a term which penalizes network complexity. The most common penalty term is the sum of squares of the network weights:

$\mathrm{F}(\mathrm{x})=\sum_{\mathrm{q}=1}^{\mathrm{Q}} \mathrm{e}_{\mathrm{q}}{ }^{\mathrm{T}} \mathrm{e}_{\mathrm{q}}+\rho \sum\left(\mathrm{w}_{\mathrm{i}, \mathrm{j}}^{\mathrm{k}}\right)^{2}$

This performance index forces the weights to be small, which produces a smoother network response. The trick with this method is to choose the correct regularization parameter $\rho$. If the value is too large, then the network response will be too smooth and will not accurately approximate the underlying function. If the value is too small, then the network will overfit.

In the same conditions, this paper used different functions to train the neural network. The results show that using the function of trainbr to train neural network can get better fitting effect and generalization ability than other training functions. Trainbr (Bayesian regulation backpropagation) is a network training function that updates the weight and bias values according to L-M optimization. It minimizes a combination of squared errors and weights, and then determines the correct combination so as to produce a network that generalizes well. The process is called Bayesian regularization. 
Bayesian regularization minimizes a linear combination of squared errors and weights. It also modifies the linear combination so that at the end of training the resulting network has good generalization qualities. See MacKay ${ }^{[33]}$ for more detailed discussions of Bayesian regularization.

This Bayesian regularization takes place within the L-M algorithm. Backpropagation is used to calculate the Jacobian $\mathrm{jX}$ of performance perf with respect to the weight and bias variables $\mathrm{X}$. Each variable is adjusted according to L-M,

$$
\begin{gathered}
\mathrm{jj}=\mathrm{jX} * \mathrm{jX} \\
\mathrm{je}=\mathrm{jX} * \mathrm{E} \\
\mathrm{dX}=-\left(\mathrm{jj}+\mathrm{I}^{*} \mathrm{mu}\right) \backslash \mathrm{je}
\end{gathered}
$$

where $\mathrm{E}$ is all errors and $\mathrm{I}$ is the identity matrix.

The adaptive value mu is increased by mu_inc until the change shown above results in a reduced performance value. The change is then made to the network, and mu is decreased by mu_dec.

The parameter mem_reduc indicates how to use memory and speed to calculate the Jacobian $\mathrm{jX}$. If mem_reduc is 1 , then trainlm runs the fastest, but can require a lot of memory. Increasing mem_reduc to 2 cuts some of the memory required by a factor of two, but slows trainlm somewhat. Higher values continue to decrease the amount of memory needed and increase the training times.

Training stops when any of these conditions occurs:

* The maximum number of epochs (repetitions) is reached.

* The maximum amount of time is exceeded.

* Performance is minimized to the goal.

* The performance gradient falls below min_grad.

* mu exceeds mu_max.

* Validation performance has increased more than max_fail times since the last time it decreased (when using validation).

The logsig (as shown in the formula 4) and purelin $(\mathrm{f}(\mathrm{x})=\mathrm{x})$ transfer functions are selected as the excitation function. The neural network topology and parameters selection of the load prediction model are shown in table 2.

Table 2. The Neural Network Topology and Parameters Setting of the Load Prediction Model

\begin{tabular}{l|l}
\hline \hline the neural network type & $\begin{array}{l}\text { a single hidden layer feed forward BP neural } \\
\text { network }\end{array}$ \\
\hline input layer node number & 6 \\
\hline output layer node number & 1 \\
\hline hidden layer node number & 16 \\
\hline the transfer function of neurons & 'logsig','purelin' (excitation function) \\
\hline learning function & $\begin{array}{l}\text { 'learngd' (the weights of gradient descent/the } \\
\text { threshold value) }\end{array}$ \\
\hline the network training function & 'trainbr' (Bayesian Regularization method) \\
\hline weights (threshold) initialization method & 'initnw' (Nguyen-Widrow method) \\
\hline the biggest training time & 5000 Epochs \\
\hline the target error & $10^{-5}$ \\
\hline \hline
\end{tabular}




\section{Analysis of Load Prediction Results}

In this paper, a business building located in Beijing, China is selected to justify the feasibility of the proposed model. The study analyzes the prediction errors between the predicted and measured results as well as the prediction errors sources.

\subsection{Building Descriptions}

As shown in figure 3, the business building is mainly used for financial administration, whose total area is 0.14957 million $\mathrm{m}^{2}$. The ground area is 0.08839 million $\mathrm{m}^{2}$ (with a large atrium), and the underground area is 0.06118 million $\mathrm{m}^{2}$. Its building shape coefficient is 0.15 . The air-conditioning is operated from 8:00 to 18:00 every day. The whole building is all for self-use offices and the staff number is around 4000 .

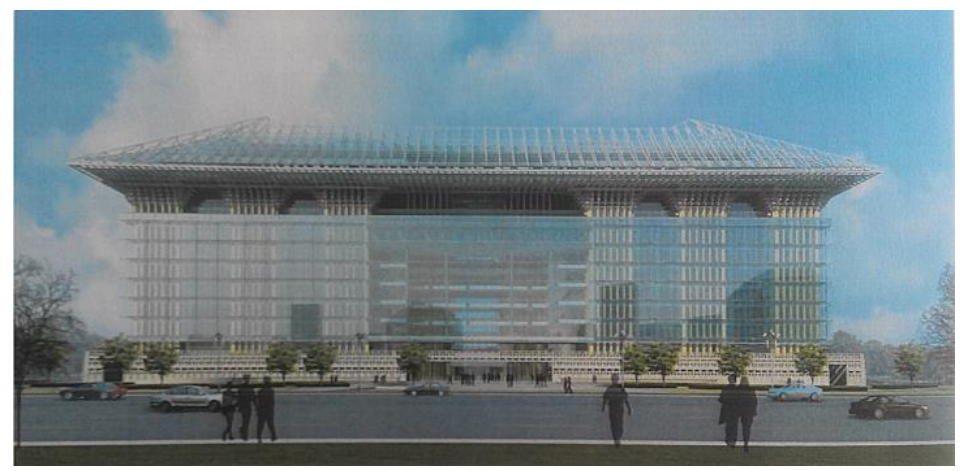

Figure 3.. The Appearance of the Business Building

\subsection{Prediction for the Next 1 Week Load}

By the constructed model, this paper makes prediction for the next 1 week hourly cooling load. In the network training courses, based on the parameters in table 2, the changing curves of each parameter for cooling load prediction are displayed in figure 4 .

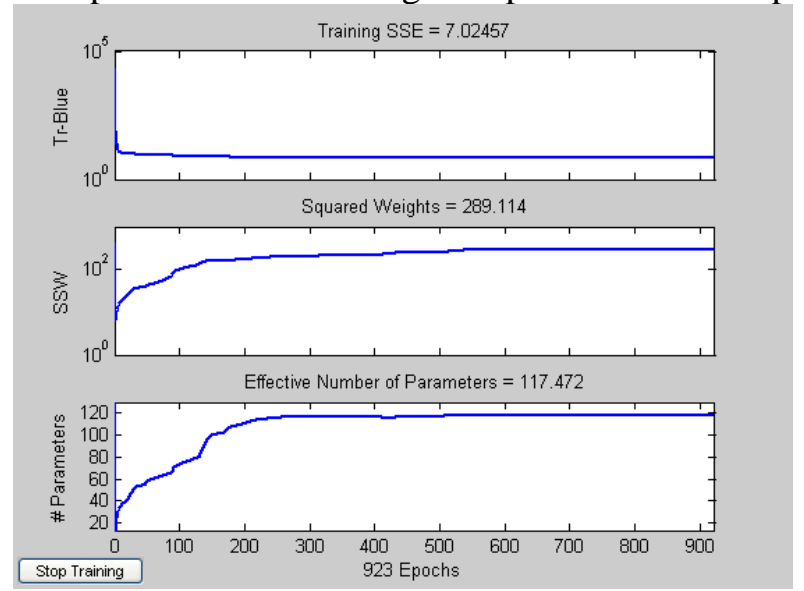

\section{Figure 4. The Shanging Process of each Parameter in the Network Training Stage}

Figure 5 shows the linear regression about the network output and the corresponding expected output. The figure shows that the tracking effect of the network output to the expected output is good and the corresponding linear correlation $\mathrm{R}=0.96369$. The collected cooling load data are mainly concentrated in the hot summer time, and the temperature and load demand change every day are similar and regular, so the neural 
network can be well fitting the nonlinear mapping relationship between the inputs and the outputs.

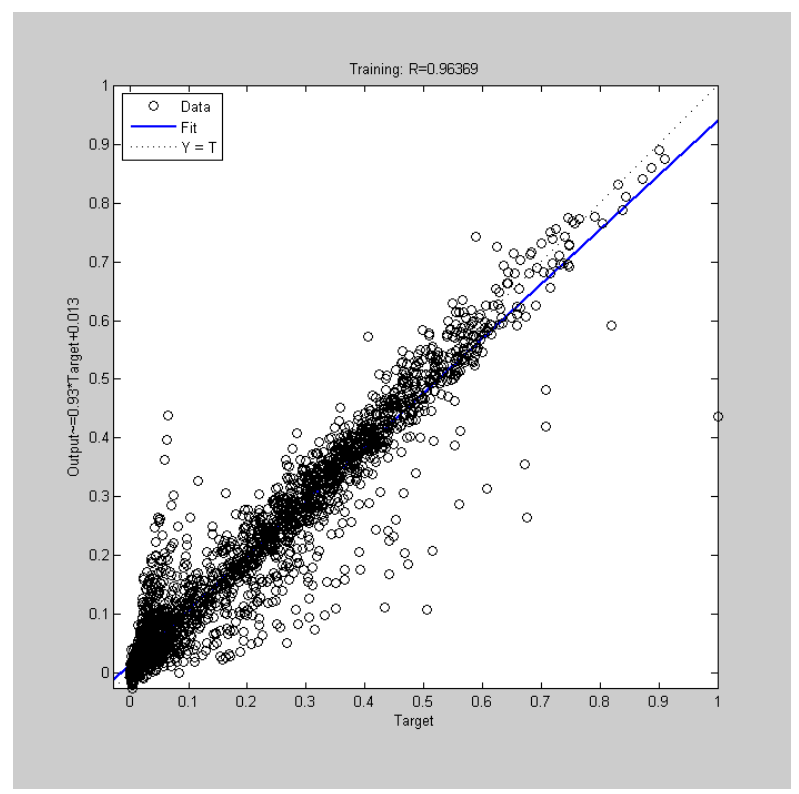

\section{Figure 5. The Linear Regression about the Network Output and the Corresponding Expected Output}

This paper uses the trained BP neural network to test the 168 sets (1 week) samples, and the test results are shown in figure 6 . It can be seen from it that although there exists a certain deviation between the prediction values and the real values, most deviation is in the range of $-0.05 \sim 0.05$. The difference between the prediction value and the real value is small, and the average prediction error (RMSE) of the test samples is $1.60 \%$. The results testify that BP prediction model is established well to describe the complex relationship between the building cooling load and input parameters. For the engineering application, the prediction error has been very well filled the bill, so the prediction method has practical value.

Outside the training sample sets, the test sample sets are the real air conditioning system data. Therefore, the test error shows that the network model and the training methods used in this paper are better, which can reflect the inherent nonlinear mapping relationship at a relatively high precision.

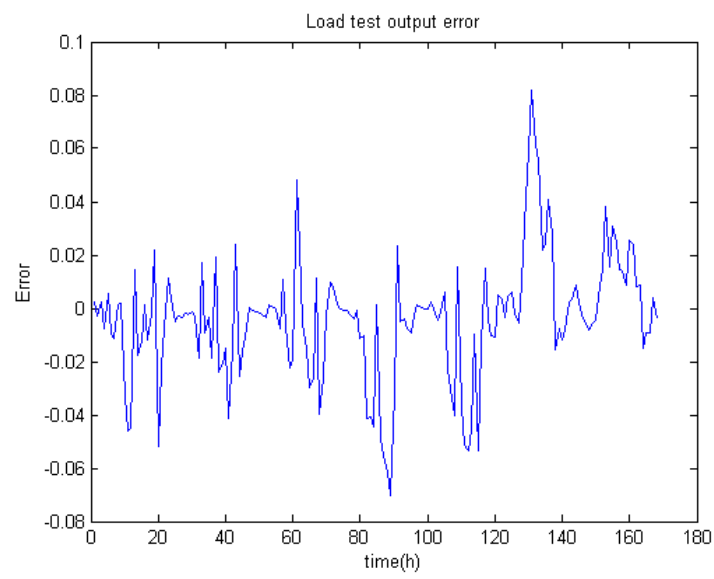

Figure 6. The Results about Cooling Load Prediction Error of 168 Sets Test Samples 
Figure 7 shows the cooling load comparison results of the calculated output load with the predicted cooling load in the test samples. In the figure, the solid line connection represents the actual output curve, and the ' + ' represents the simulation output values of the ANN model. The results show that the fitting between the actual output and the model output is good. When there is a sudden change in the hourly load, the load prediction values follow well. It shows that this model can be used to predict the cooling load of air conditioning system. Through the comparison with measured results, the practicability of the model is verified well. Through the comparison with actual output results, the practicability of the model is verified well.

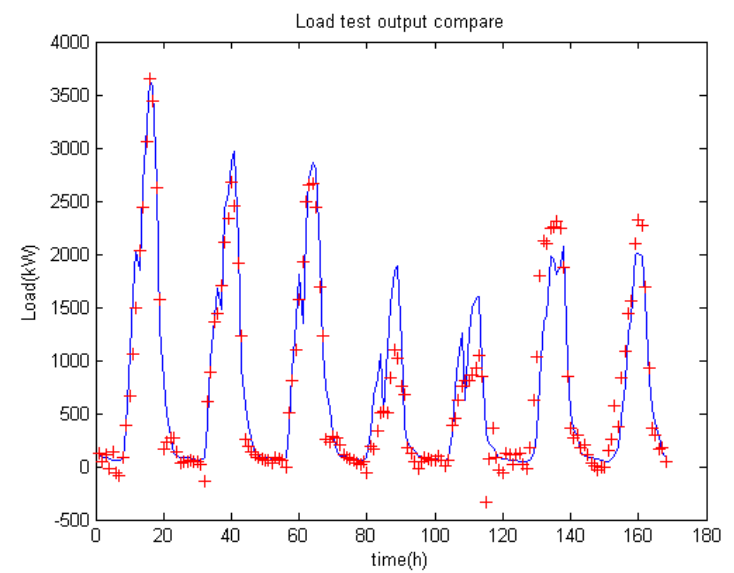

Figure 7. The Cooling Load Comparison Results of the Calculated Output Load with the Prediction Output Load

\subsection{Prediction for the Next 1day Load}

By the constructed model, this paper makes prediction for the next 1 day hourly cooling load. In the network training courses, the changing curves of each parameter for load prediction are displayed in figure 8 .

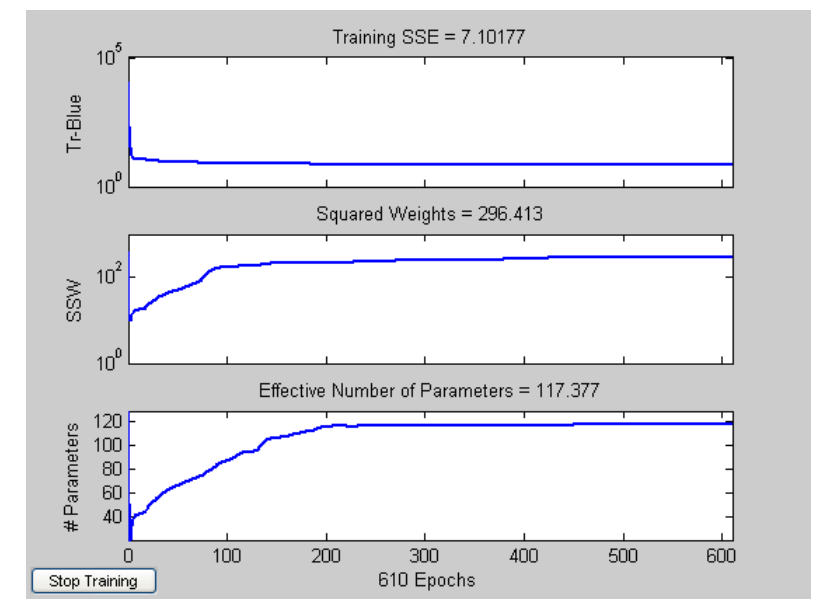

Figure 8. The Changing Process of each Parameter in the Network Training Stage

Figure 9 shows the linear regression about the network output and the corresponding expected output. The figure shows that the tracking effect of the network output to the expected output is good and the corresponding linear correlation $\mathrm{R}=0.96473$. 


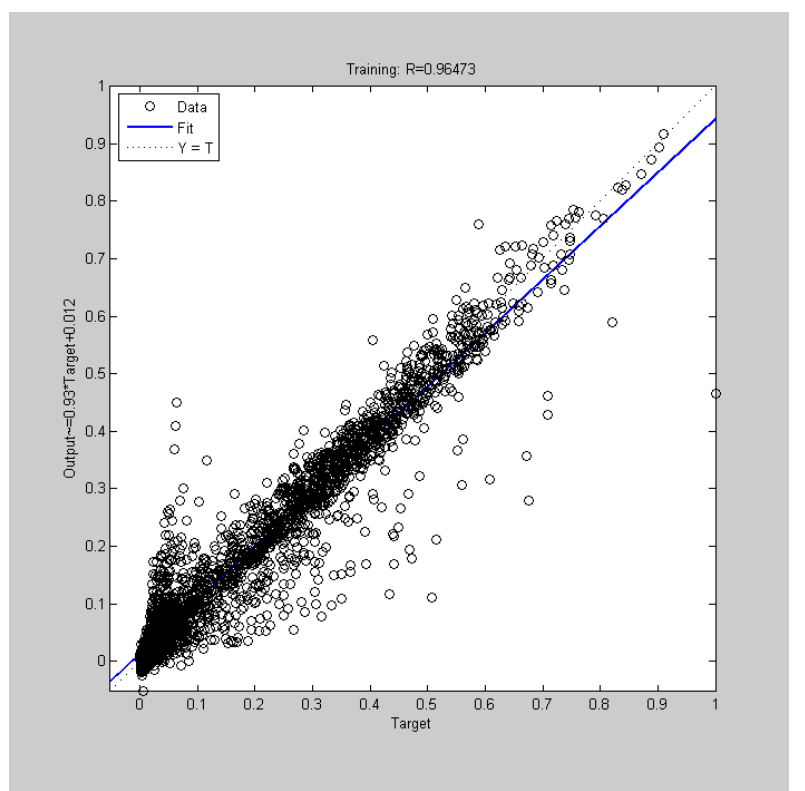

\section{Figure 9. The Linear Regression about the Network Output and the Corresponding Expected Output}

This paper uses the trained BP neural network to test the 24 sets (1 day) samples, and the test results are shown in figure 10. It is shown that although there exists a certain deviation between the prediction values and the real values, most deviation is in the range of $-0.02 \sim 0.05$.The difference between the prediction value and the real value is small, and the average prediction error (RMSE) of test samples is $1.18 \%$. For the engineering application, the prediction error has been very well filled the bill.

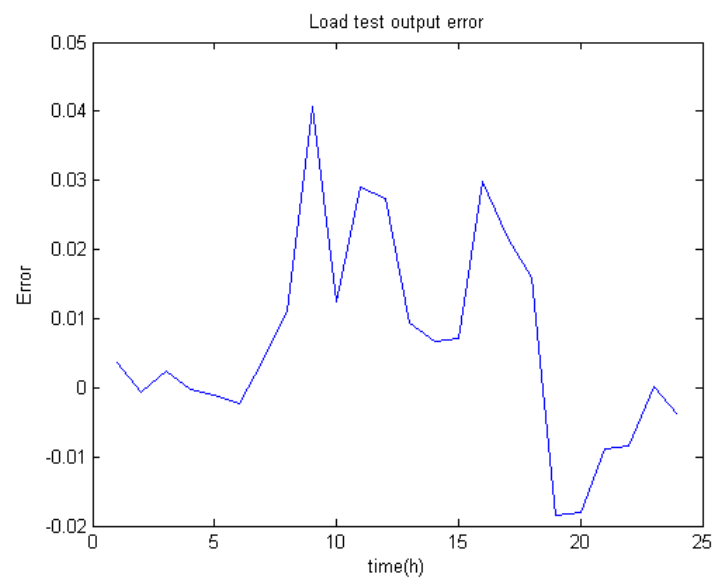

\section{Figure 10. The Results About Cooling Load Prediction Error of 24 Sets Test Samples}

Figure 11 shows the cooling load comparison results in the testing samples. The results show that the fitting between the actual output and the model output is good. When there is a sudden change in the hourly load, the load prediction values follow well. It shows that this model can be used to predict the cooling load of air conditioning systems. Through the comparison with actual output results, the practicability of the model is verified well. 


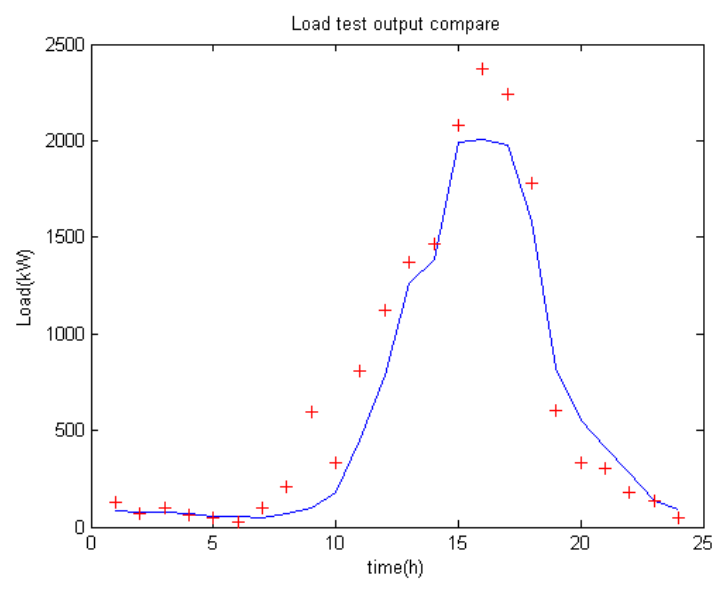

Figure 11. The Cooling Load Comparison Results of the Calculated Output Load with the Prediction Output Load

\subsection{Analysis of Prediction Errors Sources}

By the above results, it can be seen that the model constructed in this paper has a simple structure. And the required input parameters are outdoor temperature, relative humidity, speed and direction of the wind, intensity of solar radiation and indoor electrical load, etc., which can avoid the complicated calculation of unsteady heat transfer. But there are still many factors making the error exist for the prediction results. The analysis is as follows:

(1)When considering internal disturbance factors of the air conditioning system load, this paper only considers the indoor electrical load data, while the actual internal disturbance factors include the body's heat moisture quantity, etc. At the same time the number of indoor personnel and its flow should be considered.

(2)The input of the model does not consider the time delay of the cooling load caused by building thermal capacity, namely the delayed effect of meteorological parameters. In especial, the delayed effect of outdoor temperature, relative humidity and intensity of solar radiation has a great influence on the hourly load of air conditioning system, which may cause the existence of the error.

Allowing for the transition season (such as in October and November), the weather change is larger and every day or every time the cooling load demand mutation is larger. And the model proposed is not suitable for the load prediction of the transition season .So, it need to collect the transition season data in the future.

\section{Conclusion}

This paper studies the hourly load prediction of air conditioning systems and constructs a load prediction model based on BP neural networks. Taking a business building in Beijing as the actual research object, this paper had collected a total of 2928 hours of cooling load data from June to September. After the data preprocessed, for improving the generalization ability of the network, the load prediction model is trained by bayesian regularization algorithm. Then based on the constructed model, this paper makes the prediction test for the next 1 week and 1 day hourly cooling load. The results show that the prediction model can accurately predict the future hourly load of 1 week and 1 day, with the prediction error at $1.60 \%$ and $1.18 \%$ respectively. The model can be used for hourly load prediction of air conditioning systems, and the prediction accuracy is suitable. The model constructed has a simple structure, and less input parameters are needed, which can avoid complicated calculation of unsteady heat transfer. In the case of lack of the building information, it can still make a accurate prediction. This model is suitable for 
the practical engineering application and can provide a basis for optimal operation of air conditioning automatic control systems for large public buildings.

\section{Acknowledgements}

This research is sponsored by BUCEA Urban Rural Construction and Management Industry Research Development Collabration Post Graduate Training Centre and Ministry of Housing and Urban-rural Development of China (Z10025). The authors would like to thank to Beijing_Meteorological Bureau for providing meteorological data. This study is prepared as a section of Hu Chaowen's Master Thesis.

\section{References}

[1] H. L. Sun and H. P. Wang, "The Driving Factors of the Increase of the Building Energy Consumption Based on Ridge Regression”, Urban Development Studies, vol. 5, no. 20, (2013), pp. 21-24.

[2] X. T. Wu, "Considerations in energy efficient design of HAVC systems", HV\&AC, vol. 7, no. 42, (2012), pp. 1-11.

[3] M. Bida and J. F. Kreider, "Monthly-averaged cooling load calculations-residential and small commercial buildings", J Sol Energy Eng Trans ASME, vol. 4, no. 109, (1987), pp. 311-320.

[4] O. K. Vildan, "A procedure for calculating cooling load due to solar radiation: the shading effects from adjacent or nearby buildings", Energy Build, vol. 1, no. 19, (1992), pp. 11-20.

[5] Y. Yao, Z. W. Lian, S. Q. Liu and Z. J. Hou, "Hourly cooling load prediction by a combined forecasting model based on analytic hierarchy process", Int J Therm Sci., vol. 11, no. 43, (2004), pp. 1107-1118.

[6] Y. L. Wang, "Computing Method of Prediction", Science Press, (1986).

[7] W. J. Zhang, C. Yang, X. T. Han and Z. Y. Ge, "The New Development of Control Strategy for Ice Storage Air-conditioning System", HV\&AC, vol. 4, no. 24, (1994), pp. 33-35.

[8] M. Lokmanhekim and R. H. Henninger, "Computerized energy requirements analysis and heating/cooling load calculations of buildings", ASHRAE J., vol. 4, no. 14, (1972), pp. 25-33.

[9] A. Shariah, B. Tashtoush and A. Rousan, "Cooling and heating loads in residential buildings in Jordan", Energy Build, vol. 2, no. 26, (1997), pp. 137-143.

[10] T. H. S. Lao and S. M. Deng, "An evaluation of the rules of thumb for estimating cooling load for office buildings", Trans Hong Kong Inst Eng., vol. 3, no. 8, (2001), pp. 58-59.

[11] M. Bojic, F. Yik, K. Wan and J. Burnett J, "Investigations of cooling loads in high-rise residential buildings in Hong Kong”, Strojniski Vestnik/J Mech Eng., vol. 8, no. 47, (2001), pp. 491-496.

[12] O. Probst, "Cooling load of buildings and code compliance", Appl Energy, vol. 2, no. 77, (2004), pp.171-186.

[13] B. Milorad and Y. Francis, "Cooling energy evaluation for high-rise residential buildings in Hong Kong", Energy Build, vol. 4, no. 37, (2005), pp. 345-351.

[14] F. A. Ansari, A. S, Mokhtar, K. A. Abbas and N. M. Adam, "A simple approach for building cooling load estimation", Am J Environ Sci., vol. 3,no. 1, (2005), pp. 209-220.

[15] S. K. Chou and W. L. Chang, "Large building cooling load and energy use estimation", Int J Energy Res. Vol. 2, no. 21, (1997), pp. 169-183.

[16] M. S. Sodha, B. Kaur, A. Kumar and N. K. Bansal, "A comparison of the admittance and fourier methods for predicting heating/cooling loads", Solar Energy, vol. 2, no. 36, (1986), pp. 125-127.

[17] J. S. Greg and A. K. Robert, "A correlation method for predicting monthly and annual cooling loads in direct gain passive solar heated buildings", Energy Convers Manage, vol. 3, no. 29, (1989), pp. 175-187.

[18] M. Bauer and J. L. Scartezzini, "A simplified correlation method accounting for heating and cooling loads in energy-efficient buildings", Energy Build, vol. 2, no. 27, (1998), pp. 147-154.

[19] O. M. A. Rabghi and K. M. Johani, "Utilizing transfer function method for hourly cooling load calculation. Energy Convers Manage”, vol. 4, no. 38, (1997), pp. 319-332.

[20] K. W. Mui and L. T. Wong, "Cooling load calculations in subtropical climate. Build Environ.”, vol. 7, no. 42, (2007), pp. 2498-2504.

[21] D. S. He, X. Zhang and J. P. Liu, "Comparative analysis of air conditioning load prediction methods", Journal of Xi' an University of Architecture \& Technology (Natural Science Edition), vol. 38, (2006), pp. 125-129.

[22] L. Pedersen, J. Stang and R. Ulseth, "Load prediction method for heat and electricity demand in buildings for the purpose of planning for mixed energy distribution system", Energy and Buildings, vol. 40, (2008), pp. 1124-1134.

[23] D. S. He and X. Zhang. "Analysis of air conditioning load prediction by modified seasonal exponential smoothing model”, Journal of Tongji University (Natural Science), vol. 33, (2005), pp. 1672-1676.

[24] D. Robinsona, N. Campbella, W. Gaisera, K. Kabeld, A. Le-Mouelc, N. Morelb, J. Pageb, S. Stankovica and A. Stonea, "SUNtool-a new modeling paradigm for simulating and optimizing urban sustainability", Solar Energy, vol. 81, (2007), pp. 1196-1211. 
[25] M. Castilla, J. D. Álvarez, M. G. Ortega and M. R. Arahal, "Neural network and polynomial approximated thermal comfort models for HVAC systems", Building and Environment, vol. 59, (2013), pp. 107-115.

[26] I. A. Basheer and M. Hajmeer, "Artificial neural networks: fundamentals, computing, design, and application”, Journal of Microbiological Methods, vol. 43, (2000), pp. 3-31.

[27] S. Chen and S.A. Billings, "Neural networks for nonlinear dynamic system modeling and identification", International Journal of Control, vol. 56, (1992), pp. 319-346.

[28] N. Kashiwagi and T. Tobi, "Heating and cooling load prediction using a neural network system", Proceedings of the International Joint Conference on Neural Networks, (1993); New Jersey, America.

[29] Z. J. Hou, Z. W. Lian, Y. Yao and X. J. Yuan, "Cooling-load prediction by the combination of rough set theory and an artificial neural-network based on data-fusion technique", Applied Energy, vol. 83, (2006), pp. 1033-1046.

[30] E. B. Abdullatif and A. M. Mohamed, "Cooling load prediction for buildings using general regression neural networks", Energy Conversion and Management, vol. 45, (2004), pp. 2127-2141.

[31] H. F. Zheng, J. Bai and A. G. Li, "Application review of BP neutral network for prediction of cooling load in air conditioning for building", Computer Applications and Software, vol. 24, (2007), pp. 103-105.

[32] S. H. Cao, J. Z. Cao, T. Li and X. Q. Shen, "Air conditioning load prediction using neural networks based on wavelet transform", HV\&AC, vol. 35, (2005), pp. 13-17.

[33] B. Nakhi, E. Abdullatif and M. A. Mahmoud, "Cooling load prediction for buildings using general regression neural networks”, Energy Convers Manage, vol. 13-14, no. 45, (2004), pp. 2127-2141.

[34] S. A. Kalogirou, "Artificial neural networks in renewable energy systems applications: a review", Renew Sustain Energy Rev., vol. 4, no. 5, (2001), pp. 373-400.

[35] J. Yang, H. Rivard and R. Zmeureanu, "On-line building energy prediction using adaptive artificial neural networks", Energy Build, vol. 12, no. 37, (2005), pp. 1250-1259.

[36] P. A. González and J. M. Zamarreño, "Prediction of hourly energy consumption in buildings based on a feedback artificial neural network”, Energy Build, vol. 6, no. 37, (2005), pp. 595-601.

[37] "DeST Development Group in Tsinghua University, Building environmental system simulation and analysis-DeST, China Architecture \& Building Press, Beijing, (2006).

[38] Y. Jiang, "State space method for analysis of the thermal behavior of rooms and calculation of air con ditioning load", ASHRAE Trans., vol. 1, no. 88, (1981), pp. 122-132.

[39] Y. X. Zhu and Y. Jiang, "DeST-simulation tool in HVAC commissioning", Documents of IEA ANNEX 40 Workshop, no.1, (2003), pp. 1-11.

[40] X. L. Zhang, X. N. Xie, D. Yan and Y. Jiang, "Building environment design simulation software DeST(3): validation of dynamic simulation results of building thermal progress", HV\&AC, China, vol. 9, no. 34, (2004), pp. 37-50.

[41] E. S. Long and Y. Wang, "Are the relative variation rates (RVRs) approximate in different cities when the same energy-efficiency reform is taken to the same building", Build Environ., vol. 4, no. 40, (2005), pp. 453-464.

[42] M. S. Sun, W. Wang and S. E. Wan, "Design analysis of air conditioning and air distribution in the auditoria of the national theatre", HV\&AC, China, vol. 3, no. 33, (2003), pp. 1-8.

[43] L. Z. Yang and Q. L. Meng, "Influence on energy consumption of air conditioning by windows in Guangzhou's residential buildings", Xi'an Univ Architect Technol, China, vol. 1, no. 34, (2002), pp. 30-34.

[44] X. Bai, X. Xiao, S. Kun, B. Yang and L. J. Fu, “Abnormal Data Identification and Treatment in Spatial Electric Load Forecasting”, Journal of Northeast Dianli University, China, vol. 1-2, no. 33, (2013), pp. 45-50.

[45] Y. Y. Sun, "Short-Term Load Forecasting Study Based on Data Mining", Tsinghua University, Beijing, (2004).

[46] W. Dong, "Parameters Prediction and Control for Nonlinear systems Based on Neural Networks", Mechanical Industry Press, Beijing, (2008).

[47] I. Yasar, K. Akif and P. Cem, "Performance prediction for non-adiabatic capillary tube suction line heat exchanger: an artificial neural network approach", Energy Convers Manage., vol. 2, no. 46, (2005), pp. 223-232.

[48] M. T. Hagan, H. B. Demuth and M. H. Beale, "Neural Network Design”, Citic Publishing House, Beijing (2002).

[49] W. L. Jiang, Q. P. Liu and T. Liu, "Drawbacks of Neural Network Learning Algorithms and Countermeasures", Machine Tool \& Hydraulics, vol. 5, (2003), pp. 29-32.

[50] V. Cherkassky and R. Shepherd, "Regularization effect of weight initialization in back propagation networks", The World Congress on Computational Intelligence, (1998), pp. 2258-2261.

[51] W. Yan, "The Effect of Initial Parameter in Learning Algorithm of Neural Network on its Generalization 
Performance and Efficiency", Computer Engineering and Applications, vol. 23, no. 38, (2002), pp. 25-31.

[52] W. Dong, M. L. Zhang, Z. J. Jiang and M. Sun, "Neural Network Non-linear Modeling Based on Bayesian Methods", Computer Engineering and Application, vol. 11, (2005), pp. 5-11.

[53] D. C. Mackay, "Bayesian interpolation”, Neural Computation, vol. 3, no. 4, (1992), pp. 415-447.

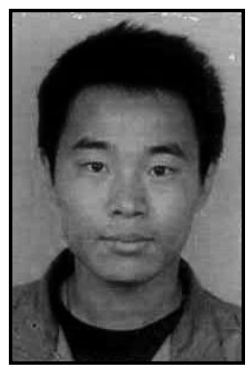

Hu Chaowen received his B.S. degree in Building Electricity and Intelligence from Hebei University of Architecture, Hebei province, China in 2013. Now he is working hard to get his M. S. degree in Control Theory and Control Engineering at Beijing University of Civil Engineering and Architecture. His research interests are mainly load prediction for buildings, building electricity, gas safety warning system constructing, as well as fault detection and diagnosis for gas regulator.

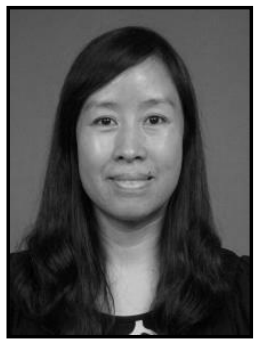

Wei Dong is presently a Professor in the School of Electrical Engineering and Information Technology at Beijing University of Civil Engineering and Architecture. She received her B.S. degree in Electrical and Automatic Engineering from Jilin University, Jilin province, China, and $\mathrm{PhD}$ in Control Science and Engineering from Beihang University, Beijing, China. Her research interests include predictive control, HVAC system modeling and control, load management, as well as fault detection and diagnosis for HVAC systems. 
International Journal of Smart Home

Vol. 9, No. 2 (2015) 